\title{
Os direitos autorais como competência profissional para a atuação do bibliotecário de referência
}

Los derechos de autor como competencia profesional del bibliotecario referencista

Copyright as a professional competence for the reference librarian

\author{
Eduardo Graziosi Silva (1), Daniel MARTínez-ÁviLA (2), Juan Carlos Fernández-Molina (3)
}

(1) Escola de Engenharia de São Carlos, Universidade de São Paulo, Av. São Carlense, 400, São Carlos - SP, Brasil, edu.gs@sc.usp.br (2) Departamento de Biblioteconomía y Documentación, Universidad Carlos III, C/Madrid, 126, 28,903 Getafe, España, dmartine@bib.uc3m.es (3) Facultad de Comunicación y Documentación, Universidad de Granada, Colegio Máximo de Cartuja, 18071 Granada, España, jcfernan@ugr.es

\begin{abstract}
Resumen
Se discuten los derechos de autor como una competencia necesaria para que el bibliotecario de referencia académica proporcione orientación sobre el uso ético y legal de la información. Se revisan los conceptos de "biblioteca académica", "servicio de referencia" y "derechos de autor" en relación con las competencias actuales de los bibliotecarios de referencia en bibliotecas académicas. Utilizando el Academic Ranking of World Universities (ARWU), se identificó un grupo oficinas de derechos de autor que representan esta nueva perspectiva para el bibliotecario de referencia y se analizaron sus páginas web con el fin de identificar los desafíos actuales y emergentes que pueden ser importantes para la implementación de estos servicios en Brasil y otros países. Se consideran competencias clave relacionadas con el servicio que la biblioteca académica y el bibliotecario de referencia proporcionan a la comunidad en relación con los derechos de autor. Se concluye que los planes de estudio de Biblioteconomía, por lo menos en Brasil, deberán revisarse para abordar estas competencias relacionadas con los derechos de autor y ajustar de forma correspondiente la educación y capacitación de los bibliotecarios de referencia.
\end{abstract}

Palabras clave: Biblioteca universitaria. Derechos de autor. Servicio de referencia. Competencias profesionales. Formación biblioteconómica.

\section{Introdução}

Com a facilidade de acesso e uso da informação proporcionada pelos meios eletrônicos, o bibliotecário de referência passou a ser não apenas o mediador entre o acervo e o usuário, mas também agregar de facto a função de orientar sobre o uso ético e legal dos recursos disponíveis. Historicamente, os aspectos mais importantes relacionados aos direitos autorais que impactaram nas bibliotecas foram: mudança da tradição oral para a tradição escrita a partir da criação da imprensa por Gutenberg em meados do século XVIII (Carboni,

\begin{abstract}
It discusses copyright as a necessary competence for the academic reference librarian in order to provide guidance on issues related to the ethical and legal use of information. It reviews the topics of "academic library", "reference service" and "copyright" in relation to the current competencies of reference librarians in academic libraries. Using the Academic Ranking of World Universities (ARWU), we identified a sample of copyright offices that represent this new perspective for the reference librarian and analyzed their websites in order to identify current and emerging issues that can be important for the implementation of these services in Brazil and elsewhere. These issues are also considered key competencies related to the service that academic library and reference librarian provide to the community in relation to copyright. We conclude that, based on the analysis of the services and competences that are present in the international libraries, the curricula of the Library and Information Science programs, at least in Brazil, need to be revised in order to address these competences related to copyright and correspondently adjust the education and training of the reference librarians.
\end{abstract}

Keywords: Academic library. Copyright. Reference service. Professional competencies. Library education.

2010; Temiño Ceniceros, 2015); o aumento da produção editorial a partir da imprensa e a custódia pelas bibliotecas, que passaram da preservação para o acesso ao acervo, sobretudo com a abertura das bibliotecas públicas norte-americanas a partir do século XIX. Com a introdução das Tecnologias de Informação e Comunicação (TICs) no século XX, houve mudanças nas instituições de ensino, dentre elas, a universidade e, consequentemente, a biblioteca universitária, especialmente em relação à sua estrutura, financiamento, serviços e públicos (Cunha, 2000; 2010). 
No século $X X I$, onde os acervos são cada vez mais híbridos, diferentes formas de acesso têm surgido. Com elas, também surgem dúvidas relacionadas ao uso da informação no ambiente digital e, consequentemente, pode haver uma maior situação de incerteza frente à garantia do acesso à informação e à proteção ao autor. Para tanto, cada vez mais está sendo exigido, na biblioteca universitária, competências e habilidades que garantam o acesso à informação sem que a Lei de Direitos Autorais seja infringida. As TICs também propiciaram novas possibilidades de produção, cópia e acesso eletrônico de documentos e migração de suportes de informação, os quais impactam diretamente no fornecimento do acesso à informação e na mudança de suporte em consonância com os direitos autorais.

Além disso, as iniciativas de ciência aberta também se configuram como um assunto em torno do qual existem dúvidas relacionadas aos direitos autorais, sendo as mais comuns aquelas relacionadas às possibilidades de uso de documentos acadêmicos (por exemplo, artigos científicos, dissertações e teses) e, mais recentemente, dados de pesquisa. Por se tratar de um assunto relativamente novo no meio acadêmico, tais dúvidas podem ser esclarecidas tanto por meio do conhecimento da legislação de direitos autorais, como também pelo contexto geral em que se desenvolve a comunicação científica. Assim, verifica-se que a carência de formação adequada sobre tais iniciativas apresenta um impacto negativo em atividades acadêmicas e de pesquisa: por um lado, a falta de informação causa insegurança a respeito do que pode ou não infringir a legislação, fazendo com que os pesquisadores adotem uma postura extremamente "defensiva", enquanto que por outro, a realização das atividades sem nenhuma precaução pode prejudicar tanto os próprios pesquisadores como suas respectivas instituições (FernándezMolina et al., 2018).

Esse contexto exige, portanto, a revisão das competências dos profissionais que nela atuam, especialmente o bibliotecário de referência, pelo fato de interagir diretamente com o público. Embora seja desejável que o bibliotecário de referência estabeleça contato com outros órgãos universitários para atender às demandas sobre direitos autorais (Gama e Garcia, 2009; Silva, 2016), oriente sobre as exceções e limitações para usos de obras protegidas, apresente fontes de informação para a resolução de questões legais (Myers, 2014) e tenha responsabilidade jurídica em relação ao usuário e à informação (Accart, 2012), esses aspetos expressos na literatura nem sempre são formalizados na prática do dia a dia da biblioteca universitária, seja pela carência de competências do bibliotecário de referência ou pela falta de planejamento ou recursos da instituição.

Assim, este trabalho reflete sobre as dificuldades encontradas pelo bibliotecário de referência que atua em biblioteca universitária para oferecer orientação em questões relacionadas aos direitos autorais. Isso posto, este estudo propõe um conjunto de competências que o bibliotecário de referência que atua em biblioteca universitária deve ter para oferecer uma correta orientação sobre direitos autorais.

\section{A evolução das competências do bibliotecário de referencia}

No início da sua atuação, a ênfase sobre a atuação do bibliotecário de referência recaía sobre suas características pessoais e pouco explorava o caráter mediador do profissional (Placer, 1968; Foskett, 1969). No que se refere às características técnicas, o foco recaía, por exemplo, sobre o zelo com o acervo, ao passo que atualmente o foco recai sobre o usuário e suas necessidades de informação. A literatura elenca mais características pessoais que profissionais, tais como: inteligência, precisão, julgamento, conhecimento profissional, confiabilidade, cortesia, desenvoltura, tato, vigilância, interesse no trabalho (Wyer, 1930 apud Bronstein, 2011), experiência, intuição, boas maneiras (Foskett, 1969), prática, raciocínio dedutivo, paciência, bom humor, cortesia, lealdade, firmeza e bom senso (McCombs, 1951). Em relação aos conhecimentos técnicos, têm-se os seguintes: aptidão na pesquisa bibliográfica, definição de procedimentos de serviços de rotina, uso de utensílios que propiciem agilidade no atendimento e fiscalização do usuário (McCombs, 1951).

Durante muito tempo, predominaram as características pessoais e técnicas, ao passo que na atualidade a literatura atualiza determinadas características técnicas. Com isso, o serviço de referência volta-se ao usuário e suas necessidades. Nesse processo, destacam-se o conhecimento das fontes de informação, o desenvolvimento de pensamento analítico para valorizar as questões do usuário e a comunicação com o público de diferentes idades, temperamentos e qualidades (Cordón García, 2002).

Atualmente, o bibliotecário de referência deve ter competências relacionadas às TICs para: implantação de serviços em rede; atendimento online via chat ou e-mail; disponibilização de formulários online para a solicitação de serviços; uso de videoconferência para realização de treinamentos, dentre outros (Jesus e Cunha, 2012). Muito embora o meio digital seja benéfico, é 
necessário considerar aspectos árduos à área, como, por exemplo, os direitos autorais. O bibliotecário de referência deve, cada vez mais, capacitar-se para orientar o usuário sobre as possibilidades de uso da informação, pois o meio digital permite sua reutilização, compartilhamento e difusão. Além disso, pode haver questões relacionadas ao uso de artigos científicos, dissertações e teses, dados de pesquisa, uso de obras intelectuais protegidas de terceiros e a propriedade intelectual das obras criadas por professores e alunos. Diante disso, podem existir conflitos entre as atividades de ensino, pesquisa e extensão pelo fato de o desenvolvimento do meio digital ter possibilitado diversas formas de uso da informação, porém, sem as devidas considerações políticoslegais, que não evoluíram na mesma velocidade que esse meio (Fernández-Molina et al., 2020). Por intermédio da oferta de orientação adequada nessas e outras possíveis questões relacionadas aos direitos autorais, o bibliotecário de referência preserva o usuário de realizar atos que possam ser considerados ilegais e salvaguarda a instituição na qual trabalha de problemas éticos em relação aos autores das obras intelectuais.

Assim, o profissional está envolvido com os meios digitais por meio do serviço de referência oferecido em plataformas virtuais e móveis. Essa mudança provocada pelas TICs modificou não somente o cotidiano do profissional e do usuário, mas principalmente o acesso à informação, que passou a oferecer maior autonomia no seu uso. Além disso, com desafios como a adequação da legislação de direitos autorais no meio digital, o bibliotecário de referência assumiu um novo papel: identifica-se a necessidade de aprimoramento das competências profissionais e aquisição de novas para atendimento das demandas atuais dos usuários. Dentre eles, tem-se o conhecimento da legislação de propriedade intelectual, com ênfase nos direitos autorais, o que pode caracterizar o profissional como assessor sobre o assunto (Fernández-Molina et al., 2011).

Nesse contexto, tem-se as diretrizes de competência em informação elaboradas por associações profissionais de Biblioteconomia e que também abordam aspectos relacionados aos direitos autorais, tais como: "Comunicação e uso ético da informação" (Lau, 2006); "A informação tem vaIor" (Association of College and Research Libraries, 2015); "A pessoa alfabetizada em informação usa informações com compreensão e reconhecimento das questões culturais, éticas, econômicas, legais e sociais em torno do uso de informações" (Bundy, 2004); "O aluno com conhecimento em informação compreende muitas das questões econômicas, legais e sociais que envolvem o uso de informações e acessa e usa informações ética e legalmente" (American Library Association, 2000); e a organização da informação de modo profissional e ético, honestidade no manuseio e disseminação da informação e capacidade de compreensão sobre direitos de outros usuários, inclusive os direitos autorais, apresentadas no pilar 5 da Society of College, National and University Libraries (SCONUL) (2013).

Diante disso, surge um programa específico de treinamento em direitos autorais, denominado copyright literacy, cujo objetivo é promover habilidades de identificação de obras intelectuais protegidas por direitos autorais, bem como seu uso ético, além de possibilitar a compreensão do fair use e fair dealing, capacitar os usuários a obter permissões ou negociar licenças e identificar violações de direitos autorais (Todorova et al., 2017).

Ademais, diante da necessidade de um suporte, assessoria e gestão especializados em direitos autorais, também surgiu a figura do copyright librarian, cuja função é oferecer informações para que o usuário tenha condições de tomar as meIhores decisões sobre direitos autorais. Algumas das atividades desenvolvidas por esse profissional são: auxiliar pesquisadores a encontrar alternativas de publicação de alta qualidade ou até gratuitas; esclarecer os legisladores acerca da sua responsabilidade sobre projetos que impactam as atividades das bibliotecas; orientar o usuário em relação ao uso legal de materiais protegidos; realizar treinamento e divulgar informações sobre direitos autorais; desenvolver e implementar políticas de direitos autorais; advogar pela aplicação da lei para o apoio às atividades acadêmicas (Vesely, 2007; Albitz, 2013; Myers, 2014).

Com o objetivo de estruturar um serviço sobre direitos autorais, algumas universidades criaram escritórios de direitos autorais a fim de centralizar as informações sobre o assunto que encontravam-se dispersas em outros setores das instituições, por exemplo, departamento jurídico e tecnologia da informação. Assim, este trabalho analisa uma amostra representativa desses escritórios.

\section{Metodologia}

As universidades foram selecionadas a partir do Academic Ranking of World Universities (ARWU), edição de 2017, que foi escolhido pelo fato de ser um dos rankings universitários de maior prestígio e apresentar uma metodologia cientificamente sólida, estável e transparente. A amostra constou das cinco primeiras universidades classificadas na lista "Top 500" nos continentes África, Ásia, Europa e Oceania. Os Estados Unidos foram selecionados separadamente por serem pioneiros na implantação de escritórios de 
direitos autorais. Quando não foram localizadas universidades suficientes para a amostra, recorreu-se à lista "501-800" do referido ranking.

A amostra de escritórios foi definida a partir de três critérios. Inicialmente definiu-se a localização do escritório como parte da biblioteca universitária; foram desconsiderados os casos para os quais se constataram a dependência do setor jurídico ou outro órgão universitário. $O$ segundo critério definiu que o escritório devia ser uma estrutura autônoma, isto é, na qual fosse possível verificar a existência de uma pessoa responsável por uma equipe e que não compartilhasse serviços com outros setores. Por fim, o último critério estabelecido foi que as informações disponíveis no site do escritório fossem suficientemente detalhadas para permitir a definição das competências necessárias para a atuação do bibliotecário de referência nesse ambiente.

A partir desses critérios, foram identificados 24 escritórios de direitos autorais, sendo 19 nos Estados Unidos, 2 no Canadá e 1 no Reino Unido, 1 na Holanda e 1 na Austrália. A relação completa com os nomes dos escritórios e seus respectivos sites encontra-se disponível no Apêndice 1.

\section{Resultados e discussão}

Os escritórios de direitos autorais encontram-se em diferentes estágios nas regiões analisadas. Enquanto que nos países anglo-saxões (Estados Unidos, Canadá, Reino Unido e Austrália) eles estão mais desenvolvidos, na Europa ainda encontra-se em desenvolvimento. $O$ estudo não identificou escritórios de direitos autorais na África, Ásia, Brasil e América Latina. Essa situação pode ser explicada pelo fato de que os primeiros escritórios foram criados nos Estados Unidos. Além disso, a consolidação dos escritórios no Canadá, no Reino Unido e na Austrália pode ser explicada pela influência dos Estados Unidos nas suas respectivas indústrias culturais e do entretenimento, além de suas legislações de direitos autorais terem aspectos comuns à legislação norte-americana.

Os diferentes estágios de desenvolvimento dos escritórios também justificam-se pelo fato das TICs terem sido introduzidas em diferentes momentos nas instituições analisadas. Conforme as TICs ofereceram novas formas de acesso e uso da informação, as bibliotecas universitárias verificaram a necessidade de implantação de um serviço voltado à garantia do uso ético dos recursos de informação em conformidade com as regras institucionais e a legislação de direitos autorais.
Assim, verificou-se que os primeiros escritórios foram compostos por equipes de diferentes órgãos universitários, as quais procuraram identificar as necessidades de informação da comunidade acadêmica e, a partir disso, buscaram desenvolver a competência em informação em direitos autorais por meio de formações extracurriculares, bem como desenvolveram sites institucionais com informações específicas sobre direitos autorais.

Observou-se, ainda, que as equipes dos escritórios possuem profissionais com outras formações além de Biblioteconomia e Ciência da Informação, com destaque para profissionais formados em Direito. Considerando-se o contexto de criação dos escritórios e que o bibliotecário ainda carece de formação adequada sobre direitos autorais (Charbonneau e Priehs, 2014; Estell e Saunders, 2016; Fernández-Molina et al., 2017; Morrison e Secker, 2017), é pertinente que as equipes sejam formadas por um grupo de profissionais atuantes em áreas do conhecimento distintas, pois desta forma a biblioteca universitária poderá oferecer suporte, assessoria e gestão adequados para as questões jurídicas apresentadas pelos usuários.

Deste modo, a amostra estudada permitiu identificar os assuntos abordados pelos escritórios em cinco temas centrais (direitos autorais e ensino; direitos autorais e pesquisa; comunicação científica e acesso aberto; comunicação científica e direitos autorais; comunicação científica e impacto da pesquisa), cujos assuntos mais explorados são apresentados na Tabela I.

\begin{tabular}{ll}
\hline Assunto & Temas mais explorados \\
\hline Direitos autorais e ensino & $\begin{array}{l}\text { Fair use/exceções aos } \\
\text { direitos autorais; obtenção de } \\
\text { permissão }\end{array}$ \\
\hline $\begin{array}{l}\text { Direitos autorais e } \\
\text { pesquisa }\end{array}$ & $\begin{array}{l}\text { Fair use/exceções aos } \\
\text { direitos autorais; plágio } \\
\text { acadêmico }\end{array}$ \\
\hline Comunicação científica e & $\begin{array}{l}\text { Conceitos básicos de acesso } \\
\text { aberto; política de acesso } \\
\text { acesso aberto } \\
\text { charge (APC) }\end{array}$ \\
\hline $\begin{array}{l}\text { Comunicação científica e } \\
\text { direitos autorais }\end{array}$ & $\begin{array}{l}\text { Direitos autorais dos } \\
\text { professores; contrato de } \\
\text { transferência de direitos } \\
\text { autorais }\end{array}$ \\
\hline Comunicação científica e & Dicas de publicação; ORCiD \\
impacto da pesquisa &
\end{tabular}

Tabela I. Assuntos abordados e temas mais explorados

Os resultados corroboram estudos anteriores que mostram maior familiaridade do bibliotecário 
com determinados temas, por exemplo, fair use, porém, ele precisa se familiarizar com temas emergentes ou ainda pouco comuns, tais como: licenciamento, acesso aberto, digitalização, licenças abertas (copyleft e Creative Commons), obras órfãs, repositórios institucionais e questões relacionadas ao ensino a distância (Estell e Saunders, 2016).

No que se refere às iniciativas de ciência aberta, destaca-se o movimento de acesso aberto. Ele tem ganhado força tanto com o aumento do preço de assinaturas das publicações científicas, que vem se tornando cada vez mais onerosa para a biblioteca universitária, bem como pelo fato de permitir tanto o acesso como a divulgação de resultados de pesquisa de uma forma mais ampla (Suber, 2012), superando, assim, algumas das exceções e limitações impostas pela legislação de direitos autorais vigente em muitos países.

Da mesma forma, o uso de conteúdos pelos professores para fins educacionais também se configura como uma das dúvidas relacionadas aos direitos autorais. Além disso, ressalta-se que tanto professores como alunos são produtores de conteúdo, porém, como o nível de conhecimento de conhecimento sobre direitos autorais dos professores (Sims, 2011; Di Valentino, 2016) não é maior que o dos estudantes (Wu et al., 2010; Datig e Russell, 2015). Questões como quais direitos de um artigo científico permanecem com o autor e quais com o editor?, quais são as permissões do editor para auto arquivamento e em quais condições?, as universidades podem obrigar os estudantes a publicar suas dissertações ou teses em acesso aberto?, existem direitos autorais ou outro tipo de proteção intelectual sobre dados de pesquisa? exigem tanto conhecimento de direitos autorais como do contexto da comunicação científica para serem respondidas (Fernández-Molina et al., 2018).

À medida em que as práticas de acesso e uso da informação vem sendo modificadas tanto pelas TICs como pelas iniciativas de acesso aberto, observam-se nos escritórios a ascensão de alguns temas, conforme demonstrado no assunto "Comunicação científica e direitos autorais". Dessa forma, verifica-se que os usuários, a fim de manterem suas práticas de publicação e visibilidade e impacto de suas pesquisas, têm demandado com mais frequência auxílio em relação à publicação científica e também em relação aos identificadores de pesquisadores, especialmente 0 ORCiD, como forma de aumentar a visibilidade dessas publicações.

Por fim, considera-se que para a aquisição, desenvolvimento ou aprimoramento dos direitos autorais como uma competência do bibliotecário de referência, é desejável que os currículos dos cursos de Biblioteconomia e Ciência da Informação abordem o assunto (Schmidt e English, 2015), especialmente em disciplinas relacionadas ao serviço de referência, assim como o profissional deve buscar aprimoramento por meio de formações extracurriculares sobre direitos autorais, tanto na área de Biblioteconomia e Ciência da Informação como em outras áreas do conhecimento.

\section{Conclusão}

Considera-se que na atualidade as competências profissionais do bibliotecário de referência, especialmente aquelas relacionadas ao uso ético e legal da informação, sobrepõem-se às competências pessoais. Ressalta-se, ainda, que a aquisição, desenvolvimento ou aprimoramento das competências profissionais voltadas à ética foram baseadas em diretrizes de competência em informação e códigos de ética elaborados por órgãos de classe e associações profissionais.

Portanto, para atender as demandas relacionadas ao uso ético e legal da informação no contexto universitário, é desejável que o bibliotecário de referência adquira, desenvolva ou aprimore as competências em direitos autorais, conforme apontam Charbonneau e Priehs (2014) e Kawooya et al. (2015). Embora a importância dos direitos autorais possa variar conforme as atividades desenvolvidas pelo profissional, eles se destacam como uma competência para sua atuação no contexto universitário (Saunders, 2020).

Por isso, espera-se que os direitos autorais sejam incorporados pelo bibliotecário de referência como uma competência que permita oferecer suporte, assessoria e gestão tanto nas atividades próprias de sua atuação como naquelas relacionadas ao uso ético e legal da informação. Nesse contexto, o profissional deve atuar como mediador entre os interesses dos autores e dos usuários tendo em vista sua missão de promover o acesso à informação de forma igualitária e justa.

\section{Referências}

Accart, J-P. Serviço de referência: do presencial ao virtual. Brasília: Briquet de Lemos, 2012.

Albitz, R. S. (2013). Copyright information management and the university library: staffing, organizational placement and authority. // The Journal of Academic Librarianship 39:5 (2013) 429-435.

American Library Association. (2000). Information literacy competency standards for higher education. Chicago: American Library Association, 2000. http://www.ala.org/ acrl/standards/informationliteracycompetency (2017-1011)

Association of College and Research Libraries. Framework for information literacy for higher education. Chicago: American Library Association, 2015. http://www.ala.org/ acrl/standards/ilframework (2017-04-20). 
Bronstein, J. (2011). The role and work perceptions of academic reference librarians: a qualitative inquiry. // Portal: Libraries and the Academy. 11:3 (2011) 1-39.

Bundy, A. Australian and New Zealand Information Literacy Framework: principles, standards and practice. Adelaide: Australian and New Zealand Institute for Information Literacy, 2004. http://adbu.fr/wp-content/uploads/2013/02/ Infolit-2ndedition.pdf (2017-10-17).

Carboni, G. Direito autoral e autoria colaborativa na economia da informação em rede. São Paulo: Quartier Latin, 2010.

Charbonneau, D. H.; Priehs, M. Copyright awareness, partnerships, and training issues in academic libraries. // The Journal of Academic Librarianship, 40:3-4 (2014), 228233.

Cordón García, J. A. Los usuarios. In: Orera, L. O. Manual de biblioteconomía. Madrid: Editorial Síntesis, 2002. P. 265287.

Cunha, M. B (2000). Construindo o futuro: a biblioteca universitária brasileira em 2010. // Ciência da Informação. 29:1 (2000) 71-89.

Datig, I.\& Russell, B. (2015). The fruits of intellectual labor: international student views of intellectual property. // College and Research Libraries, 76:6 (2015) 811-830.

Di Valentino, L. (2016). Laying the foundation for copyright policy and practice in Canadian universities. Doctoral thesis, London: University of Western Ontario, 2016.

Estell, A.; Saunders, L. Librarian copyright literacy: self-reported copyright knowledge among information professionals in the United States. // Public Services Quarterly, 12:3 (2016)., 214-227.

Fernández-Molina, J. C.; Martínez-Ávila, D.; Silva, E. G. (2020). University copyright/scholarly communication offices: analysis of their services and staff profile. // The Journal of Academic Librarianship. 46:2 (2020) 1-13.

Fernández-Molina, J. C.; Moraes, J. B. E.; Guimarães, J. A. C. (2017). Academic libraries and copyright: do librarians really have the required knowledge? // College and Research Libraries, 78:2 (2017) 241-259.

Fernández-Molina, J. C.; Silva, E. G.; Martínez-Ávila, D. (2018). Anales de VIII Conferencia Biredial-Istec 2018, Derechos de autor y ciencia abierta: el papel de la biblioteca universitaria: Lima, Octubre 22-25, 2018. Lima: Pontificia Universidad Católica del Perú, 2018. 1-14.

Fernández-Molina, J. C.; Vives-Gràcia, J.; Guimarães, J. A. C. (2011). Asesor en derechos de autor: ¿un nuevo rol del bibliotecario universitario? // Revista EDICIC. 1:4 (Oct./Dic. 2011) 49-61.

Foskett, D. J. (1969). Serviço de referência. // Serviço de informação em bibliotecas. São Paulo: Polígono, 1969. cap. 6, 90-106.

Gama, J. G. de O.; Garcia, L. G. (2009). Direito à informação e direitos autorais: desafios e soluções para os serviços de informação em bibliotecas universitárias. // Informação \& Sociedade: Estudos. 19:2 (2009) 151-162.

Jesus, D.; Cunha, M. B. (2012). Produtos e serviços da web 2.0 no setor de referência das bibliotecas. // Perspectivas em Ciência da Informação. 17:1 (2012) 110-133.
Kawooya, D.; Veverka, A; Lipinski, T. (2015). The Copyright librarian: a study of advertising trends for the period 20062013. // The Journal of Academic Librarianship. 41 (2015) 341-349.

Lau, J. (2006). Guidelines on information literacy for lifelong learning. Boca del Río: IFLA, 2006. https://www.ifla.org/files/assets/information-literacy/publications/iflaguidelinesen.pdf (2017-10-10)

McCombs, C. F. (1951). Administração e trabalho do departamento de referência. // McCombs, C. F. O departamento de referência. 2. ed Rio de Janeiro: Departamento de Imprensa Nacional, 1951. 19-32.

Morrison, C. M.; Secker, J. (2017). Understanding librarians' experiences of copyright: findings from a phenomenographic study of UK information professionals. // Library Management. 38:6/7 (2017) 354-368.

Myers, C. S. (2014). Answering copyright questions at the reference desk: a guide for academic librarians. // The Reference Librarian. 55:1 (2014) 49-73.

Placer, X. (1968). Técnica do serviço de referência. Rio de Janeiro: Associação Brasileira de Bibliotecários, 1968.

Saunders, L. Core knowledge and specialized skills in academic libraries. (2020) // College \& Research Libraries, 81:2 (2020). 288-311.

Schmidt, L.; English, M. (2015). Copyright instruction in LIS programs: report of a survey of standards in the U.S.A. // The Journal of Academic Librarianship. 41:6 (2015) 736743.

Silva, E. G. (2016). The Role of academic reference librarians in Copyright Law. // Eden, B. L. (Ed.). Envisioning our preferred future: news services, jobs, and directions. Lanham: Rowman \& Littlefield, 2016. 121-132.

Sims, N. (2011). Lies, damned lies, and copyright (mis)information: empowering faculty by addressing key points of confusion. Proceedings of the ACRL 2011 Conference: Philadelphia, PA, Mar.30-Apr.2 Chicago: Association of College \& Research Libraries. 282-296.

Society of College, National and University Libraries. The SCONUL 7 Pillars of information literacy through a digital literacy 'lens'. 2013. https://www.sconul.ac.uk/sites/default/files/documents/Digital_Lens.pdf (2018-05-26)

Suber, P. (2012). Open access. Cambridge: The MIT Press, 2012.

Temiño Ceniceros, I. (2015). El plagio en el derecho de autor. Pamplona: Civitas, 2015.

Todorova, T. et al. (2017). Information professionals and copyright literacy: a multinational study. // Library Management. 38:6/7 (2017) 323-344.

Vesely, S. A. (2007). Do you need a copyright librarian? // Internet Reference Services Quarterly. 11:4 (2007) 69-82.

Wu, H.C., Chou, C., Ke, H.R. \& Wang, M.H. (2010). College students' misunderstandings about copyright laws for digital library resources. // The Electronic Library. 30:2 (2010) 197-209.

Wyer, J. I. (1930). Reference work: a textbook for students of library work and librarians. Chicago: American Library Association, 1930.

\section{Apêndice 1: Amostra de escritórios de direitos autorais analisada}

\begin{tabular}{ll}
\hline Escritório de direitos autorais & URL \\
\hline Cambridge University (Reino Unido) & https://osc.cam.ac.uk/about-us \\
\hline Columbia University (EUA) & https://copyright.columbia.edu \\
\hline Cornell University (EUA) & https://copyright.cornell.edu \\
\hline
\end{tabular}

Silva, Eduardo Graziosi; Martínez-Ávila, Daniel; Fernández-Molina, Juan Carlos. Os direitos autorais como competência profissional para a atuação do bibliotecário de referência. // Ibersid. 14:1 (en.-jun. 2020) 79-85. ISSN 1888-0967. 


\begin{tabular}{|c|c|}
\hline Duke University (EUA) & https://library.duke.edu/about/depts/scholcomm \\
\hline Harvard University (EUA) & https://osc.hul.harvard.edu \\
\hline Leiden University (Holanda) & https://www.library.universiteitleiden.nl/research-and-publishing/copyright \\
\hline $\begin{array}{l}\text { Massachusetts Institute of } \\
\text { Technology (EUA) }\end{array}$ & https://libguides.mit.edu/c.php?g=176063\&p=3015339 \\
\hline Northwestern University (EUA) & https://www.library.northwestern.edu/research/scholarly/index.html \\
\hline Ohio State University (EUA) & https://library.osu.edu/copyright \\
\hline Pennsylvania State University (EUA) & https://libraries.psu.edu/about/departments/scholarlycommunications-and-copyright \\
\hline Princeton University (EUA) & http://library.princeton.edu/services/scholarly-communications \\
\hline Purdue University (EUA) & https://www.lib.purdue.edu/uco \\
\hline $\begin{array}{l}\text { University of British Columbia } \\
\text { (Canadá) }\end{array}$ & https://copyright.ubc.ca \\
\hline $\begin{array}{l}\text { University of California, Berkeley } \\
\text { (EUA) }\end{array}$ & http://www.lib.berkeley.edu/scholarly-communication \\
\hline $\begin{array}{l}\text { University of California, Los Angeles } \\
\text { (EUA) }\end{array}$ & $\begin{array}{l}\text { https://www.library.ucla.edu/support/copyright-datapublishing/scholarly-communication- } \\
\text { services }\end{array}$ \\
\hline $\begin{array}{l}\text { University of California, San Diego } \\
\text { (EUA) }\end{array}$ & https://ucsd.libguides.com/ScholComm \\
\hline University of Chicago (EUA) & https://www.lib.uchicago.edu/copyrightinfo \\
\hline $\begin{array}{l}\text { University of Illinois Urbana- } \\
\text { Champaign (EUA) }\end{array}$ & https://www.library.illinois.edu/scp \\
\hline University of Melbourne (Austrália) & https://copyright.unimelb.edu.au/ \\
\hline University of Michigan (EUA) & https://www.lib.umich.edu/copyright \\
\hline $\begin{array}{l}\text { University of North Carolina at Chapel } \\
\text { Hill (EUA) }\end{array}$ & https://library.unc.edu/scholcom \\
\hline University of Toronto (Canadá) & https://onesearch.library.utoronto.ca/copyright \\
\hline University of Washington (EUA) & http://www.lib.washington.edu/scholpub \\
\hline $\begin{array}{l}\text { Washington University St. Louis } \\
\text { (EUA) }\end{array}$ & https://scholarlycommunications.wustl.edu \\
\hline
\end{tabular}

Enviado: 2020-04-01. Segunda versión: 2020-05-18. Aceptado: 2020-06-03. 